\title{
PARTITIONS OF PRODUCTS
}

\author{
BY
}

DAVID PINCUS AND J. D. HALPERN

\begin{abstract}
This paper extends some applications of a theorem of Halpern and Lauchli on partitions of products of finitary trees. The extensions are to weak infinite products of dense linear orderings, and ultrafilter preservation for finite product Sacks forcing.
\end{abstract}

Introduction. The Halpern-Lauchli (HL) theorem is proved by J. D. Halpern and H. Lauchli [4]. Another proof is given by Halpern [3]. The original purpose of the theorem was to give a model for set theory in which the Boolean prime ideal theorem is true while the axiom of choice is false. This was done by J. D. Halpern and A. Levy [5].

The Laver-Pincus (LP) theorem is a reformulation of the HL theorem due independently to R. Laver (1969, unpublished) and D. Pincus (1974). In this form the theorem has been applied to finite products of dense order types (Laver, 1969, unpublished), product Sacks forcing (J. Baumgartner, 1974, unpublished), and the analytic Ramsey theory of $\omega^{\omega}$ (K. Milliken [7]). Milliken gives proofs of the Laver-Pincus theorem in [7] and [8]. He also gives a joint generalization of it and Ramsey's theorem.

This paper will strengthen the existing applications of the HL theorem as they pertain to models of set theory without the axiom of choice, products of dense linear orderings, and product Sacks forcing. In the course of the paper we will give two proofs of the LP theorem. The original proof is one which parallels that of the HL theorem. One of our proofs will proceed directly from the result of the HL theorem. The other proof will be along the lines of the original proof but will obtain a stronger result.

The paper is organized as follows. The LP theorem and other results on products of trees are stated in tree form in $\S 1$. They are restated in matrix form in $\$ 2$ and the other tree product theorems are reduced to the matrix form of the LP theorem there. The first proof of the LP theorem, the one proceeding directly from the result of the HL theorem, is given in $\S 3$.

$\$ 4$ contains a theorem which is a special case of a conjecture of Pincus [10] on a model of set theory without the axiom of choice. The results of Halpern [2], Halpern and Levy [5] and Pincus [9] suggest a way to proceed from a given model of set theory without the axiom of choice to a combinatorial theorem which implies that the Boolean prime ideal theorem holds in the model. In [10] a model is given

Received by the editors May 11, 1979 and, in revised form, December 2, 1980.

AMS (MOS) subject classifications (1970). Primary 05C55, 04A20, 03E40; Secondary 03E25, $05 C 05$. 
which satisfies the principle of dependent choice but not the full axiom of choice. The combinatorial principle which implies the Boolean prime ideal theorem in this model involves the countable ordinal numbers, $\alpha$. The case $\alpha=0$ is an easy consequence of the HL theorem. The case $\alpha=1$ is what we will prove here. It properly generalizes the HL theorem. The conjecture is open for higher $\alpha$ 's. The problem of giving a model of set theory satisfying dependent choice and the Boolean prime ideal theorem but not full choice is solved in [11]. An affirmative answer to the conjecture of this section nevertheless would still have interesting consequences. For example one could then give a model of set theory with choice in which there is a nonprincipal ultrafilter $\mu$ on the power set of $\omega$ ( $\omega$ is the set of natural numbers), a Vitali (or Banach Tarski) nonmeasurable set $V$, and a Lusin set $L$ such that no well ordering of the real numbers can be defined using $\mu, V, L$, or real numbers as parameters.

$\$ 5$ presents a generalization to weak infinite products of one of Laver's original applications of the LP theorem. The generalization is made possible by an infinite version of the LP theorem given in $\$ 1$. Otherwise the proof closely follows Laver's own (1969, unpublished). It appears with his permission.

$\$ 6$ concerns the theory of product Sacks forcing. If $L$ is the constructible universe and $s$ is a Sacks real it was known in folklore that every subset of $\omega$ in $L(s)$ includes or is disjoint from an infinite constructible subset of $\omega$. Baumgartner used the Laver-Pincus theorem to generalize this to $L\left(s_{1}, \ldots, s_{d}\right)$ where $s_{1}, \ldots, s_{d}$ are independent (product generic) Sacks reals. R. Solovay (unpublished) strengthened the folklore result by showing that any selective ultrafilter in $L$ generates a selective ultrafilter in $L(s)$. In this section we prove a selective ultrafilter version of the LP theorem and use it to obtain the corresponding result for $L\left(s_{1}, \ldots, s_{d}\right)$.

1. Tree theorems in subtree form. The subtree form of the HL theorem and its variants seem to be most convenient for applications, but the somewhat stronger but less perspicacious matrix form has been the key to the proofs.

A finitary tree is a partially ordered set $(T, \leqslant)$ (more simply $T$ ) with least element in which every element has a finite nonempty set of immediate successors and a totally ordered set of predecessors. $T(n)$, the $n$th level of $T$ is the set of elements of $T$ which have exactly $n$ predecessors. For us all trees are finitary and hence $T(n)$ is finite for all $n$.

$S \subset T$ is a strong subtree if $S$, under its inherited order, is a tree and there is a level function $f: \omega \rightarrow \omega$ satisfying $S(n) \subset T(f(n))$, and for each $n$,

$$
(\forall x \in S \cap T(n))(\forall y \in T(n+1))(\exists z \in S)[x<y \rightarrow y \leqslant z] .
$$

$S_{i} \subset T_{i}$ for $i \in I$ is a level family of strong subtrees if there is a single $f$ which is a level function for each $S_{i}, i \in I$.

TheOrem 1 (LAVer-Pincus). Let $T_{i}, i<d \in \omega$, be trees. Let

$$
\bigcup_{n \in \omega} \prod_{i<d} T_{i}(r)=\bigcup_{j<p} P_{j}
$$

for some $p \in \omega$. Then there is a level family of strong $S_{i} \subset T_{i}$ for $i<d$ and $a j<p$ such that $\cup_{n \in \omega} \Pi_{i<d} S_{i}(n) \subset P_{j}$. 
The other tree partition theorems in this section are generalizations of Theorem 1 to infinitely many trees. The most direct generalization would be to replace $d$ with $\omega$ in Theorem 1. Unfortunately using the axiom of choice one can construct a counterexample as follows: For each $i \in \omega$, let $T_{i}$ be the full binary tree. Topologize $\Pi_{i \in \omega} T_{i}(n)$ by the Tychonoff product of discrete topologies. A standard construction, involving the axiom of choice, gives for each $n$ a partition $\prod_{i \in \omega} T_{i}(n)$ $=P_{n} \cup Q_{n}$ where neither $P_{n}$ nor $Q_{n}$ has a perfect subset. It follows that neither $P_{n}$ nor $Q_{n}$ includes a set of the form $\Pi_{i \in \omega} A_{i}$ where $A_{i} \subset T_{i}(n)$ and $\left|A_{i}\right| \geqslant 2 . P=$ $\cup_{n \in \omega} P_{n}$ and $Q=\cup_{n \in \omega} Q_{n}$ form a counterexample to the $d=\omega$ generalization of Theorem 1.

A generalization of Theorem 1 to infinitely many trees must relax in some way the requirement that the $S_{i}$ be strong subtrees or the requirement that $\cup_{n} \Pi_{i \in \omega} S_{i}(n) \subset P_{j}$. Milliken [7] proposes a relaxation of the first requirement. He calls $T$ perfect if

$$
(\forall x \in T)[x \text { has at least } 2 \text { incomparable successors ]. }
$$

He conjectures that Theorem 1 holds with $d=\omega$ when the level family of strong subtrees is replaced by a level family of perfect subtrees. It is unknown whether Milliken's conjecture is true. ${ }^{1}$ Nevertheless, Milliken and others have obtained many interesting consequences.

Our own results relax the requirement that $\cup_{n \in \omega} \Pi_{i \in \omega} S_{i}(n) \subset P_{j}$. We only assert that some "tail" together with the given finite sequence is in $P_{j}$.

Theorem 2. Let $T_{i}, i \in \omega$, be trees. Let

$$
\bigcup_{n \in \omega} \prod_{i \in \omega} T_{i}(n)=\bigcup_{j<p} P_{j}
$$

for some finite $p$. Then there is a level family (with level function $f$ ) of strong subtrees $S_{i} \subset T_{i}, i \in \omega$ and $a j<p$ such that

$$
(\forall d \in \omega)(\text { Ae } n \in \omega)\left(\forall \vec{x} \in \prod_{i<d} S_{i}(n)\right)\left(\exists \vec{y} \in \prod_{i>d} T_{i}(f(n))\right)\left[\vec{x} \hat{y} \in P_{j}\right]
$$

The expression "(Ae $x \in X) \ldots$ " should be read "For all but finitely many $x \in X \ldots$ " or "For almost every $x \in X \ldots$ " The end to end join of the sequences $\vec{x}$ and $\vec{y}$ is written $\vec{x} \vec{y}$. In a slight abuse of notation this expression will be used both in the case of disjoint domains or in the case that Domain $\vec{x}=n$ and Domain $\vec{y}=m$ or $\omega$.

Theorem 2 generalizes Theorem 1 in that for any $d$ one could consider a partition which ignores $X_{i}, i \geqslant d$. If $S_{i}, i<d$ are produced as in Theorem 2 one could produce $S_{i}, i<d$ satisfying Theorem 1 by throwing away $S_{i}(n)$ for the finitely many levels $n$ which do not satisfy

$$
\left(\forall \vec{x} \in \prod_{i<d} S_{i}(n)\right)\left(\exists \vec{y} \in \prod_{i>d} T_{i}(f(n))\right)\left[\vec{x} \hat{y} \in P_{j}\right]
$$

\footnotetext{
${ }^{1} R$. Laver has recently announced a proof of what we have called Milliken's conjecture. The original formulation of this conjecture may be due to J. Baumgartner.
} 
Theorem 3 considers powers of a single tree $T$. If, in Theorem 2 , all $T_{i}$ are equal to $T$ one still cannot expect all $S_{i}$ to be equal to a fixed $S$. The problem is that equality types can separate elements. (Consider, for example, a partition of $\cup_{n \in \omega} T(n)^{\omega}$ given by $\vec{x} \in P_{0} \leftrightarrow x_{0}=x_{1}$ and $\vec{x} \in P_{1} \leftrightarrow x_{0} \neq x_{1}$.) What one says instead is that every equality type includes a more restrictive equality type in which the conclusion of Theorem 2 holds.

Let $T$ be a fixed tree, $n, N \in \omega$, and $\vec{x} \in T(n)^{N}$. Type

$$
\vec{x}=\left\{\vec{y} \in \bigcup_{m \in \omega} T(m)^{\omega}:(\forall i, j<N)\left[x_{i} \leqslant y_{i} \wedge\left(x_{i}=x_{j} \leftrightarrow y_{i}=y_{j}\right)\right]\right\} .
$$

If $\vec{x} \in T(n)^{N}$ and $\vec{y} \in T(m)^{M}$ say $\vec{x} \leqslant \vec{y} \leftrightarrow$ Type $\vec{y} \subset$ Type $\vec{x}$.

TheOREM 3. Let $T$ be a tree and $\vec{x} \in T(m)^{M}$ for some $m, M \in \omega$. Let Type $\vec{x}=$ $\cup_{j<p} P_{j}$ for some finite $p$. Then there is a strong $S \subset T$ (with level function $f$ ), $a$ $y \geqslant x$ with entries in $S$, and $a j<p$ such that

$$
(\forall d \in \omega)(\text { Ae } n \in \omega)\left(\forall \vec{w} \geqslant \vec{y}: \vec{w} \in S(n)^{d}\right)\left(\exists \vec{z} \in T(f(n))^{\omega}\right)\left[\vec{w}^{\wedge} \vec{z} \in P_{j}\right] .
$$

To see that Theorem 3 generalizes Theorem 1 consider a single tree $T$ formed from the disjoint union of the $T_{i}, i<d$ by adding a base point. $\vec{x}$ can be the sequence in $T(1)^{d}$ which picks the least element of each $T_{i}$. Notice that it is a rather strong assertion about $S$ that it is both a strong subtree and has a $\vec{y} \geqslant \vec{x}$ with entries in $S$.

2. Tree theorems in matrix form. The matrix form is the way the HL theorem was originally stated and it is the form in which it and most of its variants are proved.

$D \subset T$ is $(h, k)$ dense if

$$
(\exists x \in T(h))(\forall y \in T(h+k))[\exists z \in D][x \leqslant y \rightarrow y \leqslant z] .
$$

$x$ is called a center of $D$. Note that a minimal $(h, k)$ dense set has a unique center. A $(0, k)$ dense set is called $k$ dense. $M \subset \Pi_{i<d} T_{i}$ is an $(h, k)$ matrix if there are $(h, k)$ dense $D_{i} \subset T_{i}, i<d$ such that $\Pi_{i<d} D_{i} \subset M$. A vector of centers of the $D_{i}$ is called a center of $M$. A $(0, k)$ matrix is called a $k$ matrix.

THEOREM 2.1. Let $\cup_{n} \Pi_{i<d} T_{i}(n)=\cup_{j<p} P_{j}$ for some finite $p$. Either $P_{0}$ includes $a$ $k$ matrix for every $k$, or there is $j<p$ and $h \in \omega$ such that for every $k, P_{j}$ includes an $(h, k)$ matrix.

Notice that Theorem 2.1 follows easily from the $p=2$ case. In view of this subsequent theorems will be stated in terms of a 2 partition $P, Q$.

Proof of Theorem 1 From TheOREM 2.1. This proof is quite straightforward. However, we will present it in some detail because some interesting points arise in the corresponding argument for Theorem 6.1 in $\$ 6$.

According to Theorem 2.1 there is a single component of the partition, $P, a$ single $h$, and for each $k$ a level $m_{k}$ such that $P \cap \prod_{i<d} T_{i}\left(m_{k}\right)$ includes an $(h, k)$ matrix.

From this it follows that there is an infinite $X \subset \omega$ such that for each $m \in X$ if $s(X, m)$ is the successor in $X$ of $m$, then $P \cap \Pi_{i<d} T_{i}(s(X, m))$ includes an $(h, m+$ $1-h)$ matrix. Such an $X$ will be called a set of tree levels for the partition. 
$\Pi_{i<d} T_{i}(h)$ is finite so for some $\vec{a} \in \Pi_{i<d} T_{i}(h), Y=\{k \in X:(\exists m)[k=s(X, m) \wedge$ $P \cap \Pi_{i<d} T_{i}(k)$ includes an $(h, m+1-h)$ matrix with center $\left.\left.\vec{a}\right]\right\}$ is infinite. Notice that for $m \in Y, s(X, m) \leqslant s(Y, m)$ so $Y$ is also a set of tree levels for $p$. Furthermore the matrices from levels in $Y$ have a common center. Such a $Y$ will be called a centered set of tree levels for $p$. By changing $h$ to the least element of $Y$, (stepping up one level and cutting down matrices) one gets that $Z=Y-$ \{least element $Y$ \} is a centered set of tree levels for $P$, and furthermore $\vec{a} \in P$.

The centered set $Z$ of tree levels gives rise to a level family $S_{i} \subset T_{i}$ of strong subtrees whose common level function is the increasing enumeration of $Z$ and which satisfy $\cup_{k \in \omega} \Pi_{i<d} S_{i}(k) \subset P$. One takes $a_{i}$ as the base of $S_{i}$. Having formed $S_{i}(k) \subset T_{i}(m)$ where $m$ is the $k$ th element of $Z$ one lets $A_{i}$ be the $i$ th factor of the $(h, m+1-h)$ matrix centered at $\vec{a}$ included in $P \cap \Pi_{i<d} T_{i}(s(Z, m)) . A_{i}$ is $(h, m+1-h)$ dense so it contains a successor of every $a_{i}$ successor at level $m+1$ in $T_{i}$. In particular it contains a successor of every immediate $T_{i}$ successor of an element of $S_{i}(k)$. Thus one takes

$$
S_{i}(k+1)=\left\{b \in A_{i}:\left(\exists c \in S_{i}(k)\right)[c<b]\right\}
$$

and one will continue building full subtrees and have in addition

$$
\prod_{i<d} S_{i}(k+1) \subset P .
$$

Theorem 2 and Theorem 3 have similar matrix forms. Let $T_{i}, i \in \omega$ be trees and $t: \omega \rightarrow \omega$ be a fixed nondecreasing function. An $(h, k)$ matrix (with respect to $t$ ) is an $M \subset \Pi_{i<t(m)} T_{i}(m)$ for some $m$ such that for some $(h, k)$ dense $D_{i} \subset T_{i}(m)$, $i<t(h+k)$, every member of $\Pi_{i<t(h+k)} D_{i}$ extends to a member of $M$ (so $m \geqslant h$ $+k$ ). A strong $(h, k)$ matrix (with respect to $t$ ) is an $M \subset \Pi_{i<t(m)} T_{i}(m)$ such that for some $(h, k)$ dense $D_{i}, i<t(h+k)$,

$$
\prod_{i<t(h+k)} D_{i} \times \prod_{t(h+k)<i<t(m)} T_{i}(m) \subset M .
$$

A (strong) $(0, \mathrm{k})$ matrix is called a (strong) $k$ matrix.

THEOREM 2.2. Let $T_{i}, i \in \omega$ be trees and $t: \omega \rightarrow \omega$ be nondecreasing. If $\cup_{n} \Pi_{i<t(n)} T_{i}(n)=P \cup Q$ then either for some $h$ and all $k, Q$ includes a strong $(h, k)$ matrix or for all $k, P$ includes a $k$ matrix.

COROLlary 2.2. Under the assumptions of Theorem 2.2 if $\cup_{n} \Pi_{i<t(n)} T_{i}(n)=$ $\cup_{j<p} P_{j}$ for some finite $p$, then for some $j<p, h \in \omega$ and all $k, P_{j}$ includes an $(h, k)$ matrix.

Corollary 2.2 is an easy induction from the $p=2$ case which, in turn, is weaker than Theorem 2.2. Theorem 2 follows from Corollary 2.2 as follows. For each $n$ choose $\vec{y}_{n} \in \Pi_{i>n} T_{i}(n)$ and, given a partition of $\cup_{n} \Pi_{i \in \omega} T_{i}(n)$ define a partition of $\cup_{n} \Pi_{i<n} T_{i}(n)$ which takes $\vec{x} \in \Pi_{i<n} T_{i}(n)$ to the cell of $\vec{x} \vec{y}_{n}$. Apply Corollary 2.2 with $t(n)=n$ and form the $S_{i}$ as in the proof of Theorem 1 letting $S_{i}(k+1)$ come from an $(h, f(k)+1)$ matrix in $\Pi_{i<m} T_{i}(m)$ for some appropriate $m$. Notice that, in the statement of Theorem 2, the $\vec{y} \in \Pi_{i>d} T_{i}(f(n))$ such that $\vec{x} \hat{y} \in P_{j}$ can actually be made to come from $\Pi_{i \geqslant d} S_{i}(n)$. 
Proof of Theorem 2.2 from Theorem 2.1. Let $\cup_{n} \Pi_{i<t(n)} T_{i}(n)=P \cup Q$ as in Theorem 2.2. Assume for some $k_{0}$ that $P$ includes no $k_{0}$ matrix. Partition $\cup_{n} \Pi_{i<t\left(k_{0}\right)} T_{i}(n)$ as follows.

$$
\begin{aligned}
& \vec{x} \in Q^{*} \cap \prod_{i<t\left(k_{0}\right)} T_{i}(n) \leftrightarrow n<t\left(k_{0}\right) \vee\left(\forall \vec{y} \in \prod_{t\left(k_{0}\right)<i<t(n)} T_{i}(n)\right)[\vec{x} \hat{y} \in Q], \\
& \vec{x} \in P^{*} \cap \prod_{i<t\left(k_{0}\right)} T_{i}(n) \leftrightarrow n \geqslant t\left(k_{0}\right) \wedge\left(\exists \vec{y} \in \prod_{t\left(k_{0}\right)<i<t(n)} T_{i}(n)\right)\left[\vec{x}^{\wedge} \vec{y} \in P\right] .
\end{aligned}
$$

Theorem 1 applies to $P^{*}$ and $Q^{*}$. If $P^{*}$ includes a $k_{0}$ matrix it follows from the definition of $P^{*}$ that $P$ includes a $k_{0}$ matrix. Since we assumed otherwise $P$ cannot include $k$ matrices for all $k$. Therefore $Q^{*}$ includes $(h, k)$ matrices for some $h$ and all $k$. If $k$ is sufficiently large these matrices are at levels higher than $t\left(k_{0}\right)$. In this case the definition of $Q^{*}$ shows that each such matrix gives rise to a strong $(h, k)$ matrix in $Q$.

To obtain the matrix form of Theorem 3 one must reformulate the notion of matrix so as to take account of equality types.

Let $T$ be a tree and $t: \omega \rightarrow \omega$ be nondecreasing. If $\vec{x} \in T(h)^{t(h)}$ let Type $\vec{x}=\{\vec{y}$ $\left.\geqslant \vec{x}: \vec{y} \in \cup_{m, n} T(n)^{m}\right\}$. This represents a slight change from the meaning of Type $\vec{x}$ in $\S 1$. Let $\vec{x}_{0} \in T\left(h_{0}\right)^{t\left(h_{0}\right)}$ be fixed. $M \subset T(m)^{t(m)}$ is an $(h, k)$ matrix, in Type $x_{0}$, $h \geqslant h_{0}$ if there is an $h+k$ dense set $D \subset T(m)$ and an $\vec{x}_{1} \geqslant \vec{x}_{0}$ in $T(h)^{t(h)}$ such that every element of Type $\vec{x}_{1} \cap D^{t(h+k)}$ extends to an element of $M . M$ is a strong $(h, k)$ matrix in Type $\vec{x}_{0}$ if it contains every extension of an element of Type $\vec{x}_{1} \cap$ $D^{t(h+k)}$. A (strong) $k$ matrix is a (strong) $\left(h_{0}, k\right)$ matrix.

THEOREM 2.3. Let $T$ be a tree, $t: \omega \rightarrow \omega$ be nondecreasing, and $\vec{x}_{0}$ be a fixed element of $T\left(h_{0}\right)^{t\left(h_{0}\right)}$. If Type $\vec{x}_{0}=P \cup Q$ then either for some $h$ and all $k, Q$ includes a strong $(h, k)$ matrix or for all $k, P$ includes a $k$ matrix.

COROllary 2.3. Under the assumptions of Theorem 2.3 if Type $x_{0}=\cup_{j<p} P_{j}$ for some finite $p$, then for some $j<p, h \in \omega$, and all $k, P_{j}$ includes an $(h, k)$ matrix.

It is left to the reader to derive Theorem 3 from Corollary 2.3.

ProOF of TheOREM 2.3 From THEOREM 2.1. We may assume without loss of generality that $\vec{x}_{0} \in T(1)^{t(1)}$. Furthermore, since a matrix is a subset of some $T(m)^{t(m)}$, we may restrict attention to Type $\vec{x}_{0} \cap \cup_{n \in \omega} T(n)^{t(n)}$. For proof by contradiction, assume that $P$ does not include a $k_{0}$ matrix in Type $\vec{x}_{0}$, some $k_{0} \in \omega$. Let $N=\left|T\left(k_{0}^{+}\right)\right|$and let

$$
g: N \stackrel{\text { bijection }}{\rightarrow} T\left(k_{0}^{+}\right) .
$$

We define trees $T_{i}, i \leqslant N$, and apply Theorem 2.1 to $\cup_{n} \Pi_{i<N} T_{i}(n)$. For $i<N, T_{i}$ is the subtree of $T$ consisting of $g_{i}$, the successors of $g_{i}$, and $O_{T}$, the root of $T . T_{N}$ is the tree such that

$$
T_{N}(0)=O_{T}, \quad T_{N}(1)=\left\{f: f: t\left(k_{0}^{+}\right) \rightarrow N \text { and } g \circ f \in \operatorname{Type}\left(\vec{x}_{0}\right)\right\},
$$


and in a harmless stretching of the notion of tree we let

$$
\begin{aligned}
T_{N}(k) & =T_{N}(1) \quad \text { for } k>1 . \\
f_{1} & \leqslant T_{N} f_{2} \text { iff } f_{1}=O_{T} \text { or } f_{1}=f_{2} .
\end{aligned}
$$

(Note: $\Pi_{i \leqslant N} T_{i}(1)=\left\{(\vec{g}, f): f: t\left(k_{0}^{+}\right) \rightarrow N\right.$ and $\vec{g} \circ f \in$ Type $\left.\vec{x}_{0}\right\}$.)

Partition $\cup_{n} \Pi_{i \leqslant N} T_{i}(n)=P^{*} \cup Q^{*}$ by

$$
\begin{aligned}
& (\vec{b}, f) \in Q^{*} \text { iff every appropriate extension of } \vec{b} \circ f \text { is in } Q \text {. } \\
& (\vec{b}, f) \in P^{*} \text { iff some appropriate extension of } \vec{b} \circ f \text { is in } P \text {. }
\end{aligned}
$$

$\left(\vec{x} \in \cup_{m, n} T(n)^{m}\right.$ is an appropriate extension of $\vec{b} \circ f$ if $\vec{x} \mid t\left(k_{0}^{+}\right)=\vec{b} \circ f$.)

We show

(1) $A$ includes a 1 matrix in $\prod_{i<N} T_{i}(m) \leftrightarrow \Psi(A)$ is a $k_{0}$ matrix of Type $\vec{x}_{0}$ in $T\left(k_{0}+m\right)^{t\left(k_{0}^{+}\right)}$where $\Psi(A)=\{\vec{b} \circ f:(\vec{b}, f) \in A\}_{0}$.

Proof. First show $(\vec{b}, f) \in \prod_{i<N} T_{i}(m)$ implies $\vec{b} \circ f \in$ Type $\vec{x}_{0} \cdot \vec{g} \circ f$ is of type $\vec{x}_{0}$ by the definition of $T_{N}$. Since $(\vec{b}, f) \in \Pi_{i \leqslant N} T_{i}(m), \vec{b}_{i} \geqslant g_{i}$ for all $i$. So for all $i$

$$
(\vec{b} \circ f)_{i} \geqslant(\vec{g} \circ f)_{i} \geqslant \vec{x}_{0 i} \text {. }
$$

Furthermore if $\vec{x}_{0 i}=\vec{x}_{0 j}$ then $(\vec{g} \circ f)_{i}=(\vec{g} \circ f)_{j}$. Since $\vec{g}$ is a bijection $f_{i}=f_{j}$ and conversely so

$$
(\vec{b} \circ f)_{i}=(\vec{b} \circ f)_{j} \quad \text { iff } \quad \vec{x}_{0 i}=\vec{x}_{0 j}
$$

Suppose $A$ is a 1 matrix in $\Pi_{i \leqslant N} T_{i}(1)$. (We take $m=1$ for ease of exposition and with only slight loss of generality.) Then $A=\Pi_{i<N} T_{i}(1)$. Let $D=T\left(k_{0}^{+}\right)$. We show $\left\{\vec{g} \circ f: f \in T_{N}(1)\right\}=D^{t\left(k_{0}^{+}\right)} \cap$ Type $\vec{x}_{0}$.

The previous proof gives us

$$
\Psi(A)=\left\{\vec{g} \circ f: f \in T_{N}(1)\right\} \subset D^{t\left(k_{0}^{+}\right)} \cap \text { Type } \vec{x}_{0} .
$$

Let $\vec{x} \in D^{t\left(k_{0}^{+}\right)} \cap$ Type $\vec{x}_{0}$. Let $f=\vec{g}^{-1} \circ \vec{x}$. Then $\vec{g} \circ f=\vec{x} \in$ Type $\vec{x}_{0}$. So $f \in$ $T_{N}(1)$, so $\vec{x}=\vec{g} \circ f \in \Psi(A)$. So

$$
D^{t\left(k_{0}^{+}\right)} \cap \text { Type } \vec{x}_{0} \subset \Psi(A) .
$$

Now suppose $\Psi(A)$ is a $k_{0}$-matrix of Type $\vec{x}_{0}$ in $T\left(k_{0}+m\right)^{t\left(k_{0}^{+}\right)}$. Let $(\vec{b}, f) \in A$. Then $\{\vec{b}\}$ is 1 dense in $\Pi_{i<N} T_{i}(m)$. So $\left\{(\vec{b}, f): f \in T_{N}(1)\right\}$ is a 1 dense matrix included in $A$.

Show

(2) $A$ is an $(h, k)$ matrix in $\Pi_{i \leqslant N} T_{i}(m)$ implies that $\Psi^{*}(A)$, the set of all appropriate extensions of elements of $\Psi(A)$, includes a strong $\left(k_{0}+h, k\right)$ matrix of Type $\vec{x}_{0}$ in $T\left(m+k_{0}\right)^{t\left(m+k_{0}\right)}$.

Proof. Suppose $A$ is as above and assume $h>0$. Then there exists $\vec{a} \in$ $\Pi_{i<N} T_{i}(h)$ and $(h, k)$ dense sets $D_{i} \subset T_{i}$ with $a_{i}$ as the center such that for some $f \in T_{N}(1)$

$$
\prod_{i<N} D_{i} \times\{f\} \subset A .
$$

$D_{i}$ is $(h, k)$ dense in $T_{i}$ means that $D_{i}$ is $\left(h+k_{0}, k\right)$ dense in $T$. Let $D=\cup_{i<N} D_{i}$. Extend $D$ in a conservative way to an $\left(k_{0}+h+k\right)$ dense subset of $T\left(m+k_{0}\right)$. Let 
$D_{1}=T\left(m+k_{0}\right)-\left\{x: \exists i, x \geqslant a_{i}\right\}$. Then $D \cup D_{1}$ is an $\left(k_{0}+h+k\right)$ dense subset of $T\left(m+k_{0}\right)$ and

$$
\left(D \cup D_{1}\right)^{t\left(k_{0}^{+}\right)} \cap \operatorname{Type}(\vec{a} \circ f) \subseteq D^{t\left(k_{0}^{+}\right)} \cap \operatorname{Type}(\vec{a} \circ f) .
$$

Show $D^{t\left(k_{0}^{+}\right)} \cap \operatorname{Type}(\vec{a} \circ f) \subseteq \Psi(A)$ :

For $\vec{x} \in D^{t\left(k_{0}^{+}\right)} \cap \operatorname{Type}(\vec{a} \circ f)$ define $g^{\prime}: N \rightarrow D$ such that $g_{i}^{\prime} \geqslant a_{i}$ and $g_{f(j)}^{\prime}=x_{j}$, all $j \in t\left(k_{0}^{+}\right)$. These conditions are consistent since $x_{j} \geqslant a_{f(J)}$. Then $\vec{g}^{\prime} \in \Pi_{i<N} D_{i}$ and $\vec{g}^{\prime} \circ f \in \operatorname{Type}(\vec{a} \circ f)$ (since $\vec{a}$ and $\vec{g}^{\prime}$ are both 1-1) and $\vec{g}^{\prime} \circ f=\vec{x}$. So we have $\left(D \cup D_{1}\right)^{t\left(k_{0}^{+}\right)} \cap \operatorname{Type}(\vec{a} \circ f) \subseteq \Psi(A)$. From this it follows that every extension in $T\left(m+k_{0}\right)^{t\left(m+k_{0}\right)}$ of elements of $\left(D \cup D_{1}\right)^{t\left(k_{0}+h+k\right)} \cap \operatorname{Type}(\vec{a} \circ f)$ is in $\Psi^{*}(A)$. Furthermore $\vec{a} \circ f \geqslant \vec{x}_{0}$, so $\Psi^{*}(A)$ includes a strong $\left(k_{0}+h, k\right)$ matrix in $T\left(m+k_{0}\right)^{t\left(m+k_{0}\right)}$.

Proof of Theorem. Since $P$ does not contain a $k_{0}$ matrix of Type $\vec{x}_{0}$ it follows that $P^{*}$ does not include a 1 matrix. Therefore by Theorem 1 there is an $h$ such that for all $k, Q^{*}$ includes an $(h, k)$ matrix. Therefore by (2) and the definition of $Q^{*}$, there is an $h$ such that for all $k, Q$ includes a strong $(h, k)$ matrix of Type $\vec{x}_{0}$.

This concludes the proof of Theorem 2.3 from Theorem 2.1. Notice that Theorems 1, 2, 3, 2.2, and 2.3 have all been proven modulo a proof of Theorem 2.1.

3. Proof of Theorem 2.1 from the HL theorem. As previously mentioned, Milliken gives a proof of Theorem 2.1 in [8]. Thus we will content ourselves with presenting the basic ideas of the derivation of Theorem 2.1 from the HL theorem.

TheOrem (Halpern-Lauchli). Let $T_{i}, i<d<\omega$, be trees, and let $\Pi_{i<d} T_{i}=$ $\cup_{j<p} P_{j}$ for some $p \in \omega$. Then either $P_{0}$ includes a $k$ matrix for every $k$, or there is $j<p$ and $h \in \omega$ such that for every $k, P_{j}$ includes an $(h, k)$ matrix.

Notice that the Laver-Pincus theorem differs from the Halpern-Lauchli theorem in that the latter is concerned with partitions of the full products of the trees whereas the former is concerned with partitions of the union of products of the levels.

In stating our principles we will limit ourselves to length 2 sequences of trees $T_{1}$, $T_{2}$ in order to ease their digestion by the reader. The principles themselves are not dependent on the length of the sequence and in fact hold even if the sequences are infinite. On the other hand the presentation is not eased by limiting the partitions to 2-partitions. Consequently we will allow arbitrary finite partitions $\vec{Q}$ (a partition, for us, will be a sequence of sets rather than a family of sets).

Domination Principle (Simple Version). If $B_{1}, C_{1} \subset T_{1}$ and $B_{2}, C_{2} \subset T_{2}$ and every element of $B_{i}$ has a successor in $C_{i}$, for $i=1,2$ (write $B_{i} \leqslant C_{i}$ ) and $\vec{Q}$ partitions $C_{1} \times C_{2}$, then there is a partition $\vec{Q}^{\prime}$ of $B_{1} \times B_{2}$ with the same length as $\vec{Q}$ such that for all $h, k, \alpha$, if $\vec{Q}_{\alpha}^{\prime}$ includes an $(h, k)$ matrix then so does $\vec{Q}_{\alpha}$ (i.e., $\vec{Q}$ is as good as $\vec{Q}^{\prime}$ ).

Proof. Let $f_{1}: B_{1} \rightarrow C_{1}$ and $f_{2}: B_{2} \rightarrow C_{2}$ such that $x \leqslant f_{j}(x)\left(\right.$ all $\left.x \in B_{j}, j=1,2\right)$. Define $\vec{Q}^{\prime}$ by $\vec{Q}_{\alpha}^{\prime}=\left\{\left(x_{1}, x_{2}\right):\left(f_{1}\left(x_{1}\right), f_{2}\left(x_{2}\right)\right) \in \vec{Q}_{\alpha}\right\}$ for all $\alpha$ in the domain of $\vec{Q}$. It is an easy matter to verify that $\vec{Q}$ is as good as $\vec{Q}^{\prime}$. 
Domination PRINCIPLe (EXTENDED Version). Let $\left\{B_{i}\right\}_{i \in \omega}$ be a sequence of subsets of $T_{1},\left\{C_{i}\right\}_{i \in \omega}$ a sequence of subsets of $T_{2}$ such that for all $i<j, B_{i} \leqslant B_{j}$ and $C_{i} \leqslant C_{j}$. Let $B \subset T_{1}, C \subset T_{2}$ such that $B \leqslant \cup_{i} B_{i}, C \leqslant \cup_{i} C_{i}$.

If $\vec{Q}$ is a partition of $\cup_{i}\left(B_{i} \times C_{i}\right)$ then there is a partition $\vec{Q}^{\prime}$ of $B \times C$ such that $\vec{Q}$ is as good as $\vec{Q}^{\prime}$.

Proof. For each $i$, let $f_{1}^{(i)}, f_{2}^{(i)}$ be partial maps from $B, C$ respectively to $B_{i}, C_{i}$ respectively such that the domain of $f_{1}^{(i)},\left(f_{2}^{(i)}\right)$ is the largest subset of $B,(C)$ which is dominated by $B_{i},\left(C_{i}\right)$ and such that $x \leqslant f_{1}^{(i)}(x)$ all $x \in \operatorname{Dom} f_{1}^{(i)}\left(y<f_{2}^{(i)}(y)\right.$ all $\left.y \in \operatorname{Dom} f_{2}^{(i)}\right)$. Let $f^{(i)}$ be the map on $B \times C$ induced by $\left\langle f_{1}^{(i)}, f_{2}^{(i)}\right\rangle$ and let $\left\{\left\langle x_{n}, y_{n}\right\rangle\right\}_{n \in \omega}$ be an enumeration of $B \times C\left(x_{n}\right.$ denotes the 1st cordinate of the $n$th point, $y_{n}$ denotes the second). We define a sequence of subsets of $\omega$ by recursion. Let $S_{n}=\left\{i \in S_{n-1}: f^{(i)}\left(x_{n}, y_{n}\right) \in \vec{Q}_{L_{n}}\right\}$ where $S_{-1}=\omega$ and $L_{n}$ is chosen (take $L_{n}$ to be the least such) so that $S_{n}$ is infinite. In this way we get a sequence $S_{0} \supseteq S_{1}$ $\supseteq \ldots$ of infinite sets such that if $i, j \in S_{n}$ then $f^{(i)}\left(\left\langle x_{n}, y_{n}\right\rangle\right)$ and $f^{(j)}\left(\left\langle x_{n}, y_{n}\right\rangle\right)$ are in the same part of $\vec{Q}$. Define $\vec{Q}^{\prime}$ by $\left\langle x_{n}, y_{n}\right\rangle \in \vec{Q}_{L}^{\prime}$ if $f^{(i)}\left(\left\langle x_{n}, y_{n}\right\rangle\right) \in \vec{Q}_{L}$ for some (equiv. all) $i \in S_{n}$. We show that $\vec{Q}$ is as good as $\vec{Q}^{\prime}$. Suppose $A_{1} \times A_{2} \subset Q_{L}^{\prime}$ where $A_{j}$ is $(h, k)$ dense in $T_{j}, j=1,2$. Let $n$ be such that each point of $A_{1} \times A_{2}$ occurs among the first $n$ points in the enumeration of $B \times C$. Then $f^{(i)}(\langle x, y\rangle) \in Q_{L}$ all $i \in S_{n}$, all $x \in A_{1}$, all $y \in A_{2}$ i.e., $\left\langle f_{1}^{(i)}(x), f_{2}^{(i)}(y)\right\rangle \in Q_{L}$ all $i \in S_{n}$, all $x \in A_{1}$, all $y \in A_{2}$. Since $x \leqslant f_{1}^{(i)}(x)$ and $y \leqslant f_{2}^{(i)}(y)$ we have $f_{1}^{(i)}\left[A_{1}\right]$ is $(h, k)$ dense in $T_{1}$ and $f_{2}^{(i)}\left[A_{2}\right]$ is $(h, k)$ dense in $T_{2}$. Hence $f_{1}^{(i)}\left[A_{1}\right] \times f_{2}^{(i)}\left[A_{2}\right]$ is an $(h, k)$ matrix included in $\vec{Q}_{L}$. Thus $\vec{Q}$ is as good as $\vec{Q}^{\prime}$.

For application of the domination principle, extended version, take $T_{1}(i)$ for $B_{i}, T_{1}$ for $B, T_{2}(i)$ for $C_{i}$ and $T_{2}$ for $C$. We obtain that any partition of $\cup_{n}\left(T_{1}(n) \times T_{2}(n)\right)$ is as good as some partition of $T_{1} \times T_{2}$. Now the HalpernLauchli theorem gives the conclusion of Theorem 2.1.

4. Finite matrix versions and a model of set theory. The HL theorem was originally applied in exhibiting a model for set theory in which the Boolean prime ideal theorem is true while the axiom of choice is false. This application does not use the version of the theorem stated in \$3. Instead it uses a finite version, analogous to the finite version of Ramsey's theorem. We state and prove a finite version of Theorem 2.3 .

Theorem 4. Let $T$, $t$, and $\vec{x}_{0}$ be as in Theorem 3. Fix $p, k \in \omega$. There is an $m \in \omega$ such that whenever Type $\vec{x}_{0} \cap T(m)^{t(m)}=\bigcup_{j<p} P_{j}$ there are $j<p$ and $h<m$ such that $P_{j}$ includes an $(h, k)$ matrix.

Proof (Assuming Corollary 2.3). If Theorem 4 is false then for some $p, k$ every Type $\vec{x}_{0} \cap T(m)^{t(m)}$ has a partition into $p$ pieces which fails to include an $(h, k)$ matrix. Putting these partitions together to a single partition of Type $\vec{x}_{0} \cap$ $\cup_{m} T(m)^{l(m)}$ gives a contradiction to Corollary 2.3.

In [10] Pincus gives a model of set theory which satisfies the principle of dependent choice and the linear ordering theorem (every set can be linearly ordered) but does not satisfy full choice. He conjectures that this model satisfies the 
Boolean prime ideal theorem. If this is true, a related model with choice would have the definability properties given in the introduction to this paper.

We state below a combinatorial conjecture involving the countable ordinals $\alpha$ which would imply the Boolean prime ideal theorem in the model. We then prove the $\alpha=1$ case from Theorem 4 .

Functions of order $\alpha$ are defined by induction on the countable ordinals, $\alpha$. A function of order 0 is a map from $\omega$ to 2. A function of order $\alpha+1$ is a map from $\omega$ into the set of functions of order $\alpha$. A function of limit order $\beta$ is a function on $\beta$ whose value at $\alpha<\beta$ is a function of order $\alpha$. The Cohen conditions of [10] are finite systems of equations (henceforth called systems) among such functions. A precise formulation of this notion is set forth below.

Let 0 and 1 be terms of order -1 . For each countable ordinal $\alpha$ single out an infinite set of function symbols of order $\alpha$. A term is 0,1 , or a finite sequence beginning with a function symbol. If $S \neq 0,1$ is a term of order 0 or $\alpha+1$ then $S n, n \in \omega$ is a term of order $\alpha-1$. If $S$ is a term of limit order $\beta$ then $S \alpha, \alpha<\beta$ is a term of order $\alpha$. All terms are generated from function symbols by the above rules. A system $(U, \equiv)$ (more simply $U$ ) is a finite set of terms together with an equivalence relation between them. The following rules express the idea that equivalent terms are those connected by an equality and inequivalent terms are those connected by an inequality.

(a) If $S \in U$, so is every nonzero initial segment of $S$.

(b) If $S \equiv T$ then $S$ and $T$ have the same order.

(c) If $f$ and $g$ are distinct function symbols then $f \neq z$.

(d) $0 \neq 1$, and if $S \in U$ has order -1 then $S \equiv 0$ or $S \equiv 1$.

(e) If $S \equiv T$ and for some sequence $W, S^{\wedge} W \in U$ then $T^{\wedge} W \in U$ and $S^{\wedge} W \equiv$ $T^{\wedge} W$.

$U$ strongly extends $V$ if $U \subset V$ and $\equiv_{v}$ restricts to $\equiv_{{ }_{u}}$ on $U$. If $F$ is a set of function symbols $U \mid F$ is the system formed by restricting $\equiv_{{ }}$to the set of terms beginning with function symbols from $F$ (together with 0 and 1 ). $V \leqslant U$ if $U$ strongly extends some system $V^{*}$ which is obtained from $V$ by a 1-1 order preserving substitution of function symbols in its terms. An $\alpha$ system is a system all of whose function symbols have order $\alpha$. An $\alpha$ type is an $\alpha$ system with exactly one function symbol. If $\tau$ is an $\alpha$ type and $f$ is a function symbol of $U f$ satisfies $\tau$ when $\tau \leqslant U \mid\{f\}$.

Let $U_{n}, n \in \omega$, be an increasing sequence of $\alpha$ systems. If $f$ is a function symbol of $U_{n}, f^{+}$is the set of all function symbols $g$ of $U_{n+1}$ such that $g$ satisfies the type $U_{n} \mid\{f\}$. A set of function symbols $M$ of $U_{m}$ is an $n$ matrix when $U_{n+1}\left|f^{+}=U_{m}\right| M$. $f$ is the base of $M$.

Types and systems, modulo equivalence under 1-1 order preserving substitutions, are partially ordered by $\leqslant$. Recall that a subset $D$ of a partially ordered set is dense when every element of the set bears $\leqslant$ to an element of $D$.

Conjecture. For each countable ordinal $\alpha$ there is a dense set $D$ of systems. $D$ together with the dense set $D_{0}$ of types occurring in $D$ satisfy the following.

Let $\tau \in D_{0}$ and let $U_{n}, n \in \omega$, be an increasing sequence from $D$. There is an 
$m \in \omega$ such that whenever $\left\{f \in U_{m}: f\right.$ satisfies $\left.\tau\right\}=P \cup Q$ either $P$ or $Q$ includes an $n$ matrix, $n<m$, whose base satisfies $\tau$.

Proof of THE CONJECTURE FOR $\alpha=1$. Let $D$ be the set of 1 systems $U$ satisfying the following.

(1) There are $N_{0}, N_{1} \in \omega$ such that $f p q \in U$ exactly when $f \in U, p<N_{1}$ and $q<N_{0}$.

(2) If $S \not z T$ are terms of order $i \in 2$ then $S p \not z T p$ for some $p \in N_{i}$. This says that the equivalence class of $S$ is completely determined by the function $p \mapsto[S p]$ from $N_{i}$ into the order $i-1$ equivalence classes.

(3) Every function from $N_{i}$ into the order $i-1$ equivalence classes is realized as one of the functions $p \rightarrow[U p]$. (See (2) above.)

Let $U_{n}, 1 \leqslant n \in \omega$, be a sequence from $D$. Without loss of generality it can be assumed that the given type $\tau$ has the form $U_{i} \mid\{f\}$. Let $T$ be the tree whose $n$th level is the set of functions from $N_{0}\left(U_{n}\right)$ to 2. Let $t: \omega \rightarrow \omega$ be defined by $t(n) \leqslant N_{1}\left(U_{n}\right) . x \in T(n)$ corresponds to $[S(x)] \in U_{n}$ where $S(x) q \equiv x(q)$ for all $q<N_{0} . \vec{x} \in T(n)^{t(n)}$ corresponds to the unique function symbol $f$ of $U_{n}$ satisfying $f p \equiv S(x) p$ for all $p<N_{1}$. This correspondence carries the type $\tau$ to a Type $\vec{x}_{0}$ for some $\vec{x}_{0} \in T(1)^{t(1)}$. Similarly it carries $n$ matrices of $U_{n}$ to $n, 1$ matrices of Type $\vec{x}_{0} \cap T(n)$. Thus this case of the conjecture is reduced to Theorem 4 .

5. Products of the rational order type. Laver's original application of the LP theorem is the following where $\eta$ is the order type of the set $\mathbf{Q}$ of rational numbers.

Theorem (Laver, 1969, unPublished). Let $\mathbf{Q}^{d}=\cup_{j<p} P_{j}$. There are $X_{i} \subset \mathbf{Q}$, $i<d$, of type $\eta$ and $a j<p$ such that

$$
\left(\text { Ae } x_{0} \in X_{0}\right)\left(\text { Ae } x_{1} \in X_{1}\right) \cdots\left(\text { Ae } x_{d-1} \in X_{d-1}\right)\left[\vec{x} \in P_{j}\right] \text {. }
$$

This section is devoted to obtaining a generalization of Laver's theorem to weak infinite powers of $\mathbf{Q}$. Theorem 2 plays approximately the same role in the generalization as Theorem 1 plays in obtaining Laver's theorem.

$\mathbf{Q}^{\omega}$, the weak infinite power of $\mathbf{Q}$, is the set of all $\vec{x} \in \mathbf{Q}^{\omega}$ which are eventually 0 . It follows immediately from Laver's theorem that if $\mathbf{Q}^{\omega}=\cup_{j<p} P_{j}, d \in \omega$, and $\vec{u} \in \mathbf{Q}^{\underline{\omega}}$ then there are $j<p$ and $X_{i} \subset \mathbf{Q}, i<d$, of type $\eta$ such that

$$
\left(\text { Ae } x_{d-1} \in X_{d-1}\right)\left(\text { Ae } x_{d-2} \in X_{d-2}\right) \cdots\left(\text { Ae } x_{0} \in X_{0}\right)\left[\vec{x}^{\wedge} \vec{u} \in P_{j}\right] \text {. }
$$

Theorem 5 is in the direction of picking $X_{i}, i \in \omega$ and $j<p$ which works for each $d$ and some $U_{d}$. What actually occurs is that there is a finite set $U_{d}$ of possible vectors $\vec{u}$.

TheOREM 5. Let $\mathbf{Q}^{\underline{\omega}}=\cup_{j<p} P_{j}$. There are $X_{i}, i \in \omega$, of type $\eta$, finite $U_{d} \subset \mathbf{Q}^{\underline{\omega}}$, $d \in \omega$, and $j<p$ such that

$$
(\forall d \in \omega)\left(\text { Ae } x_{d-1} \in X_{d-1}\right) \cdots\left(\text { Ae } x_{0} \in X_{0}\right)\left(\exists \vec{u} \in U_{d}\right)\left[\vec{x}^{\wedge} \vec{u} \in P_{j}\right] .
$$

It would be interesting to know if $U_{d}$ can be assumed to be a singleton. It would also be interesting to know if the order reversal is necessary i.e., if the last line could be

$$
(\forall d \in \omega)\left(\operatorname{Ae} x_{0} \in X_{0}\right) \cdots\left(\operatorname{Ae} x_{d-1} \in X_{d-1}\right)\left(\exists \vec{u} \in U_{d}\right)\left[\vec{x}^{\wedge} \vec{u} \in P_{j}\right] .
$$


Proof. Fix a partition $\mathbf{Q}^{\underline{\omega}}=\cup_{j<p} P_{j}$. Trees $T_{i}, i \in \omega$, will be constructed by induction. Assume that levels $T_{i}(m)$ have been constructed for $i<n, m<n$. Also assume that a set $U_{x} \subset \mathbf{Q}$ of type $\eta$ has been associated to each $x \in T_{i}(m)$. The following facts will be preserved in the induction.

(a) If $m<i, T_{i}(m)$ consists of a single nonrational point. If $i \leqslant m, T_{i}(m)$ is a set of rationals.

(b) If $x$ is rational in $T_{i}(m)$ then $x \in U_{x}$.

(c) If $x_{1} \leqslant x_{2}$ in $T_{i}$ then $U_{x_{2}} \subset U_{x_{1}}$.

(d) If $x_{1} \in T_{i_{1}}$ and $x_{2} \in T_{i_{2}}$ for $i_{1} \neq i_{2}$ then $U_{x_{1}} \cap U_{x_{2}}=\varnothing$.

(e) If $x \in T_{i}(m), m<n-1$, is rational, $x$ has exactly 2 immediate successors, $x^{-}$ and $x^{+}$in $T_{i}(m+1)$.

(f) If $y_{1} \in U_{x^{-}}$and $y_{2} \in U_{x^{+}}$then $y_{1}<_{\mathbf{Q}} x<_{\mathbf{Q}} y_{2}$. It follows that if $x_{1}<_{\mathbf{Q}} x_{2}$ and $x_{1}$ is $T_{i}$ incomparable with $x_{2}$ then $y_{1}<_{\mathbf{Q}} y_{2}$ for all $y_{1} \in U_{x_{1}}, y_{2} \in U_{x_{2}}$.

(g) If $x_{i} \in T_{i}(m), i \leqslant m$ and $\overrightarrow{0}$ is the 0 sequence then for some $j<p$

$$
\left(\text { Ae } y_{m} \in U_{x_{m}}\right) \cdots\left(\text { Ae } y_{0} \in U_{x_{0}}\right)\left[\vec{y} \wedge \overrightarrow{0} \in P_{j}\right] \text {. }
$$

(h) Let $\vec{x}$ and $j$ be as in (g). Let $q \leqslant m, v \geqslant x_{q}$ in $T_{q}\left(m_{q}\right)$ and $y_{i} \geqslant x_{i}$ in $T_{i}\left(m_{i}\right)$ for $q \leqslant i \leqslant m$. Also let the $m_{i}$ be weakly decreasing with $m_{m}=m$. Then

$$
\left(\forall w \in U_{v}\right)\left(\operatorname{Ae} z_{q-1} \in U x_{q-1}\right) \cdots\left(\text { Ae } z_{0} \in U_{x_{0}}\right)\left[\vec{z}^{\wedge} w^{\wedge} \vec{y}^{\wedge} \overrightarrow{0} \in P_{j}\right] .
$$

The construction of $T_{i}(n), i \leqslant n$, proceeds as follows. For $x \in T_{i}(n-1), i<n$, let $U x^{+}=\{y \in U x: x<y\}$ and $U x^{-}=\{y \in U x: y<x\}$. Similarly let $U_{n}=\mathbf{Q}$. Finitely many successive applications of Laver's theorem with $d=n+1$ produces $\eta$ type sets $V_{x^{+}} \subset U_{x^{+}}, V_{x^{-}} \subset U_{x^{-}}$and $V_{n} \subset U_{n}$ with the following property. For every $n+1$ vector whose $i$ th term has the form $V_{x^{ \pm}}$or $V_{n}$, where $x \in T_{i}(n-1)$ when $i<n$, there is a $j<p$ such that

$$
\left(\text { Ae } y_{n} \in U_{n}\right)\left(\text { Ae } y_{n-1} \in U_{n-1}\right) \cdots\left(\text { Ae } y_{0} \in U_{0}\right)\left[\vec{y} \wedge \overrightarrow{0} \in P_{j}\right] \text {. }
$$

It can be assumed, by cutting down $V_{n}$ and the $V_{x^{ \pm}}$'s, that $V_{n}$ is disjoint from each $V_{x^{ \pm}}$. Properties (a), .., (g) will automatically hold if the $x^{ \pm}, x_{n}, U_{x^{ \pm}}$and $U_{x_{n}}$ are chosen in such a way that $x^{ \pm} \in U_{x^{ \pm}} \subset V_{x^{ \pm}}, x_{n} \in U_{x_{n}} \subset V_{n}$, the $U_{x^{ \pm}}$and $U_{x_{n}}$ have type $\eta$, and $U_{x_{n}}$ is assigned to the nonrational $z \in T_{n}(m), m<n$.

Assume that $x^{ \pm}$(or $x_{n}$ ) and $U_{x^{ \pm}}$(or $U_{x_{n}}$ ) have been defined for $x \in T_{i}(n-1)$ (or $T_{n}$ ) where $i>q$. Assume further that $\left(\mathrm{h} q^{\prime}\right)$ holds for all $q^{\prime}>q$ where:

(h $q^{\prime}$ ) Let $m \leqslant n$ and $\vec{U}$ be an $m$ vector in which $U_{i}$ is $U_{x}$, some $x \in T_{i}(m)$ (or $V_{x^{ \pm}}$some $x \in T_{i}(n-1)$, if $\left.m=n\right)$. Let $m_{i}, q^{\prime} \leqslant i \leqslant m$, weakly decrease to $m$. Let $v \in U_{q^{\prime}} \cap T_{q^{\prime}}\left(m q^{\prime}\right)$ and $y_{i} \in U_{i} \cap T_{i}\left(m_{i}\right), q^{\prime}<i \leqslant m$. If $j<p$ is as in (g) for $\vec{U}$ (or as in the definition of the $V_{x^{ \pm}}$if $m=n$ ) then

$$
(\forall w \in U v)\left(\operatorname{Ae} z_{q-1} \in U_{q-1}\right) \cdots\left(\operatorname{Ae} z_{0} \in U_{0}\right)\left[\vec{z}^{\wedge} w \vec{y}^{\wedge} \overrightarrow{0} \in P_{j}\right] .
$$

$x^{ \pm}$(or $x_{n}$ ) and $U_{x^{ \pm}}\left(\right.$or $U_{x_{n}}$ ) will be defined so as to satisfy $(\mathrm{h} q)$. For definiteness assume that $i<n$ and $x^{+}$must be defined. The point of the argument is that each particular $\vec{U}, \vec{y}$ pair necessitates throwing away finitely many elements of $V_{x^{+}}$. The resulting $U_{x^{+}}$differs finitely from $V_{x^{+}}$so it has type $\eta \cdot x^{+}$is an arbitrary member of the $U_{x^{+}}$so constructed. 
Let $m_{i}, q \leqslant i \leqslant m, \vec{U}$, and $\vec{y}$ be given as in (h $q^{\prime}$ ). If $m_{q+1} \leqslant n-1$ then no cutting down of $V_{x^{+}}$is necessary because by the inductive assumption of (h) for $n-1$

$$
(\forall w \in U x)\left(\operatorname{Ae} z_{q-1} \in U_{q-1}\right) \cdots\left(\operatorname{Ae} z_{0} \in U_{0}\right)\left[\vec{z} \hat{w} \hat{y} \hat{y} \overrightarrow{0} \in P_{j}\right] .
$$

If $m_{q+1}=n$ then taking $v=y_{q+1}$ one has by $(\mathrm{h} q+1)$

$$
\left(\operatorname{Ae} z_{q} \in U_{q}\right)\left(\operatorname{Ae} z_{q-1} \in U_{q-1}\right) \cdots\left(\operatorname{Ae} z_{0} \in U_{0}\right)\left[\vec{z} \hat{y} \hat{y} \overrightarrow{0} \in P_{j}\right] .
$$

Thus if $U_{x^{+}}$avoids the finitely many bad members of $U_{q}$ one can replace (Ae $z_{q} \in U_{q}$ ) with $\left(\forall w \in U_{x^{+}}\right)$. Therefore $x^{+}$and $U_{x^{+}}$can be defined for $x \in$ $T_{q}(n-1)$ so as to satisfy $(\mathrm{h} q)$. Hence when $q=0$, (h) is satisfied.

Carry out the above definition of the $T_{i}$ satisfying (a), ., (h) for each $n$. It is easy to see that $\left(a^{\prime}\right), \ldots,\left(f^{\prime}\right)$ can be stated for the whole sequence where the primed statement can be obtained from the unprimed one by replacing $U_{x}$ for $x \in T_{i}$ with $\operatorname{Succ}(x)=\left\{y \in T_{i}: x \leqslant y\right\}$. The statements (g) and (h) can be replaced by $\left(\mathrm{g}^{\prime}\right)$.

Let $\vec{x} \in \Pi_{i \in \omega} T_{i}(n)$. There is a $j<p$ such that

$$
\begin{aligned}
&\left(\forall m_{n} \geqslant n\right)\left(\forall y_{n} \in T_{n}\left(m_{n}\right) \cap \operatorname{Succ}\left(x_{n}\right)\right) \\
&\left(\forall m_{n-1} \geqslant m_{n}\right)\left(\forall y_{n-1} \in T_{n-1}\left(m_{n-1}\right) \cap \operatorname{Succ}\left(x_{n-1}\right)\right) \cdots \\
&\left(\forall m_{0} \geqslant m_{1}\right)\left(\forall y_{0} \in T_{0}(m) \cap \operatorname{Succ}\left(x_{0}\right)\right)\left[\vec{y} \overrightarrow{0} \in P_{j}\right] .
\end{aligned}
$$

Partition $\cup_{n \in \omega} \Pi_{i \in \omega} T_{i}(n)$ by sending $x$ to the $P_{j}$ of $\left(\mathrm{g}^{\prime}\right)$. By Theorem 2 there are strong $S_{i} \subset T_{i}$ (with level function $f$ ) and a single $j<p$ such that

$$
(\forall d \in \omega)(\text { Ae } n \in \omega)\left(\forall x \in \prod_{i<d} S_{i}(n)\right)\left(\exists y \in \prod_{i>d} T_{i}(f(n))\right)\left[\vec{x} \hat{y} \in P_{j}\right] .
$$

Let $X_{i} \subset \mathbf{Q}$ be the set of rationals in $S_{i} . X_{i}$ has type $\eta$. To show, for example, that $X_{i}$ is densely ordered consider $x_{1}<_{\mathbf{Q}} x_{2}$ in $X_{i}$. Considering the various cases of $T_{i}$ comparability of $x_{1}$ and $x_{2}$ one concludes that the $S_{i}$ successor of either $x_{1}^{+}$or $x_{2}^{-}$is between $x_{1}$ and $x_{2}\left(\operatorname{see}\left(\mathrm{f}^{\prime}\right)\right)$.

If $d \in \omega, \Pi_{i>d} T_{i}(f(d))$ is finite because all but finitely many $T_{i}(f(d))$ are singletons. For $\vec{y} \in \Pi_{i>d} T_{i}(f(d))$ let $\vec{u}(\vec{y}) \in \mathbf{Q}^{\omega}$ be the sequence defined by $u_{j}(\vec{y})$ $=y_{j+d}$ for $j \leqslant f(d)-d$ and $u_{j}(\vec{y})=0$ otherwise. Set $U_{d}=\{\vec{u}(\vec{y}): \vec{y} \in$ $\left.\Pi_{i>d} T_{i}(f(d))\right\}$.

It is claimed that

$$
\begin{aligned}
&\left(\forall z_{d-1} \in X_{d-1}: \text { level } z_{d-1} \geqslant d\right)\left(\forall z_{d-2} \in X_{d-2}: \text { level } z_{d-2} \geqslant \text { level } z_{d-1}\right) \cdots \\
&\left(\forall z_{0} \in X_{0}: \text { level } z_{0} \geqslant \text { level } z_{1}\right)\left(\exists \vec{u} \in U_{d}\right)\left[\vec{z} \vec{u} \in P_{j}\right] .
\end{aligned}
$$

This implies the desired

$$
\left(\text { Ae } z_{d-1} \in X_{d-1}\right) \cdots\left(\text { Ae } z_{0} \in X_{0}\right)\left(\exists \vec{u} \in U_{d}\right)\left[\vec{z}^{\hat{u}} \vec{u} \in P_{j}\right] .
$$

To prove the claim let $z_{0}, \ldots, z_{d-1}$ have decreasing levels above $d$ in the $S_{i}$ (i.e. above $f(d)$ in the $\left.T_{i}\right)$. Let $x_{i}$ be the predecessor of $Z_{i}$ in $S_{i}(d)$. Let $\vec{y} \in \Pi_{i>d} T_{i}(f(d))$ be such that $\vec{x} \vec{y} \in P_{j}$. It follows from $\left(\mathrm{g}^{\prime}\right)$ that $\vec{z} \hat{u}(y) \in P_{j}$. (Set $m_{i}=f(d)$, $d \leqslant i \leqslant f(d)$, and use the given $Y_{i} \in T_{i}\left(m_{i}\right)$.) 
6. Selective ultrafilters and product sacks forcing. We will be concerned with nonprincipal ultrafilters on the power set of $\omega$. Such an ultrafilter can be thought of as a finitely additive 2 valued measure, $\mu$, on subsets of $\omega$ such that $\mu(\{n\})=0$ when $n \in \omega$ and $\mu(\omega)=1$. We will often use the terms "small" and "large" to denote sets of $\mu$-measure 0 and 1 respectively. The following is one of several equivalent definitions of selective ultrafilter.

Definition ([1]). $\mu$ is selective if for every decreasing sequence $X_{0} \supset X_{1} \supset X_{2}$ $\supset$. . of large sets there is a strictly increasing function $f$ from $\omega$ to $\omega$ with large range such that $(\forall n)\left[f(n+1) \in X_{f(n)}\right]$.

We refer the reader to [6] for set theory in general and for the notions of forcing, Sacks forcing, and product forcing in particular. We assume that Gödel's constructible universe, $L$, is the ground model but everything done here would work equally well over any universe satisfying the axioms of set theory with dependent choice. As is customary we identify real numbers with subsets of $\omega$ through the binary decimal correspondence. The small ambiguity of this correspondence will not concern us here.

Let $\mu \in L$ be an ultrafilter relative to $L$. If $r \notin L$ is a real number, the family of $\mu$-large sets retains the property of not containing 0 and being closed under finite intersection relative to $L(r)$. It follows that $\mu$ generates a 2 valued measure (a filter) on a Boolean subalgebra of $P(\omega)^{L(r)}$. There is no reason in general to expect that $\mu_{r}$ will be an ultrafilter on $P(\omega)^{L(r)}$, and indeed it usually is not. For example if $r$ is a random or a Cohen real, $\mu_{r}$ is undefined on $r$. A Sacks real is, of course, a minimal constructible degree but even such degrees do not necessarily have any $\mu_{r}$ 's which are ultrafilters. If $r$ is a Silver or Gregorieff real, [1], $\mu_{r}$ is defined on $r$ but undefined on some other reals in $L(r)$. (Consider the real $t$ such that $n \in t \leftrightarrow$ $|r \cap n|$ is even.)

R. Solovay, and possibly others, have proven that $\mu_{s}$ is an ultrafilter when $s$ is a Sacks real and $\mu$ is selective. In fact $\mu_{s}$ is a selective ultrafilter relative to $L(s)$. Our purpose here is to generalize Solovay's result to $d$ independent (product generic) Sacks reals. Those unfamiliar with the theory of product forcing might wonder why this is a problem. Consider the case of 2 independent Sacks reals, $s_{0}$ and $s_{1}$. The theory of product forcing says that $s_{0}$ is $P$ generic over $L$ and $s_{1}$ is $P$ generic over $L\left(s_{0}\right)$ where $P$ is the set of perfect closed subsets of $P(\omega)$ (Sacks conditions) in $L$. The problem is that $P$ is not the set of Sacks conditions relative to $L\left(s_{0}\right)$ so that $s_{1}$, while being Sacks over $L$, may not be Sacks over $L\left(s_{0}\right)$.

In the following theorem $\mu_{s_{0}, \ldots, s_{d-1}}$ is the filter generated by $\mu$ in $L\left(s_{0}, \ldots, s_{d-1}\right)$.

TheOREM 6. Let $S_{i}, i<d$, be independent Sacks reals. Let $\mu \in L$ be a selective ultrafilter relative to $L$. Then $\mu_{s_{0}} \ldots, s_{d-1}$ is a selective ultrafilter relative to $L\left(s_{0}, \ldots, s_{d-1}\right)$.

Theorem 6 depends on the following selective ultrafilter version of Theorem 1 .

TheOREM 6.1. Let $\mu$ be a selective ultrafilter and let $T_{i}, i<d$, be trees. Partition $\cup_{n \in \omega} \Pi_{i<d} T_{i}(n)=\cup_{j<p} P_{j}$. There is a level family $S_{i} \subset T_{i}, i<d$, with common level function $f$ and $j<p$ such that $\cup_{n \in \omega} \Pi_{i<d} S_{i}(n) \subset P_{j}$ and Range $f$ is $\mu$-large. 
We will be interested only in the $p=2$ case of Theorem 6.1. The proof of the general case from the $p=2$ case is not difficult even if it is also not absolutely immediate.

Proof of Theorem 6 From Theorem 6.1. It will be shown that every real in $L\left(s_{1}, \ldots, s_{d-1}\right)$ either includes or is disjoint from a $\mu$-large real in $L$. Our argument for this from Theorem 6.1 is essentially due to Baumgartner who obtained that every real of $L\left(s_{0}, \ldots, s_{d-1}\right)$ either includes or is disjoint from an infinite real in $L$. Baumgartner used Theorem 1.

The given real is $t\left(s_{0}, \ldots, s_{d-1}\right)$ where $t$ is a term in the appropriate forcing language. It suffices to show that if the product condition $\left(U_{0}, \ldots, U_{d-1}\right) H$ " $t\left(s_{0}^{\prime}, \ldots, s_{d-1}^{\prime}\right)$ is a real," then some larger (in the componentwise reverse inclusion order) condition $\left(W_{0}, \ldots, W_{d-1}\right) H^{\prime \prime} t\left(s_{0}^{\prime}, \ldots, s_{d-1}^{\prime}\right)$ includes or is disjoint from a $\mu$-large $a \in L$."

Trees $T_{i}, i<d$, whose nodes are perfect subsets of $P(\omega)$ (Sacks conditions) will now be defined in $L . T_{i}(0)=\left\{R_{i}^{*}\right\}$ where $\left(U_{0}, \ldots, U_{d-1}\right)<\left(U_{0}^{*}, \ldots, U_{d-1}^{*}\right)$ where $\left(U_{0}^{*}, \ldots, U_{d-1}^{*}\right)$ decides " $0 \in t\left(s_{0}, \ldots, s_{d-1}\right)$." If the $T_{i}(n)$ have been defined the $T_{i}(n+1)$ will satisfy:

(a) Every node of $T_{i}(n)$ will have 2 incomparable (i.e. pairwise disjoint) successors in $T_{i}(n+1)$.

(b) Every $\left(V_{0}, \ldots, V_{d-1}\right) \in \prod_{i<d} T_{i}(n+1)$ decides " $n+1 \in t\left(s_{0}, \ldots, s_{d-1}\right)$."

(c) Each $V \in T_{i}(n+1)$ has radius less than $1 / 2^{n+1}$.

There is no problem producing nodes satisfying (a) and (c). One then steps through them in $\left(2^{n+1}\right)^{d}$ steps and makes them satisfy (b).

Partition $\cup_{n \in \omega} \Pi_{i<d} T_{i}(n)=P \cup Q$ by saying

$$
\begin{aligned}
& \left(V_{0}, \ldots, V_{d-1}\right) \in P \cap \prod_{i<d} T_{i}(n) \leftrightarrow\left(V_{0}, \ldots, V_{d-1}\right) \Vdash “ n \in t\left(s_{0}, \ldots, s_{d-1}\right) . " \\
& \left(V_{0}, \ldots, V_{d-1}\right) \in Q \cap \prod_{i<d} T_{i}(n) \leftrightarrow\left(V_{0}, \ldots, V_{d-1}\right) \Vdash “ n \notin t\left(s_{0}^{\prime}, \ldots, s_{d-1}^{\prime}\right) . "
\end{aligned}
$$

Let $S_{i} \subset T_{i}, i<d$, and $f$ be as in Theorem 6.1. Assume, for definiteness, that $\cup_{n \in \omega} \Pi_{i<d} S_{i}(n) \subset P$. Let $W_{0}, \ldots, W_{d-1}$ be the conditions obtained by fusion on the $S_{0}, \ldots, S_{d-1}$ respectively, i.e.

$$
W_{i}=\bigcap_{n \in \omega} \bigcup S_{i}(n)
$$

Since $S_{i}(n) \subset T_{i}(f(n))$ it follows from $\Pi_{i<d} S_{i}(n) \subset P$ that

$$
\left(\bigcup S_{0}(n), \bigcup S_{1}(n), \ldots, \bigcup S_{d-1}(n)\right) \Vdash “ f(n) \in t\left(\dot{s}_{0}, \ldots, \dot{s}_{d-1}\right) . "
$$

Therefore $\left(W_{0}, \ldots, W_{d-1}\right) \nVdash$ "Range $f \subset t\left(\dot{s}_{0}, \ldots, \dot{s}_{d-1}\right)$." Since $f \in L$ and Range $f$ is $\mu$ large this is what is needed. If one had assumed $\cup_{n \in \omega} \Pi_{i<d} S_{i}(n) \subset Q$ one would similarly have obtained $\left(W_{0}, \ldots, W_{d-1}\right) \Vdash$ "Range $f \cap t\left(\dot{s}_{0}, \ldots, \dot{s}_{d-1}\right)$ $=\varnothing$.

From the fact that every real of $L\left(s_{0}, \ldots, s_{d-1}\right)$ includes or is disjoint from a large real of $L$ it easily follows that $\mu_{s_{0}, \ldots, s_{d-1}}$ is an ultrafilter relative to $L\left(s_{0}, \ldots, s_{d-1}\right)$. To see that it is selective relative to $L\left(s_{0}, \ldots, s_{d-1}\right)$ it helps to remark that if $h: \omega \rightarrow L$ is in $L\left(s_{0}, \ldots, s_{d-1}\right)$ there is a $g: \omega \rightarrow L^{<\omega}$ in $L$ such that 
for all $n, h(n) \in g(n)$. This is a standard argument due to Sacks [12] for a single Sacks real and presents no problems in the product case. One builds in $L$ the trees $T_{i}, i<d$, as in the above argument demanding that each $\left(V_{0}, \ldots, V_{d-1}\right) \in$ $\Pi_{i<d} T_{i}(n)$ decides the value of $t\left(\dot{s}_{0}, \ldots, \dot{s}_{d-1}\right)$ (now a term for the function $h$ ). Fusion over the $T_{i}$ produces $\left(W_{0}, \ldots, W_{d-1}\right)$ which forces $h(n)$ to have one of at most $\left(2^{n}\right)^{d}$ values. $g(n)$ is the set of these values.

In $L\left(s_{0}, \ldots, s_{d-1}\right)$ let $X_{0} \supset X_{1} \supset X_{2} \supset \ldots$ be a decreasing sequence of large sets as in the definition of selective. Let the function $h \in L\left(s_{0}, \ldots, s_{d-1}\right)$ be given by $h(n)=Y$ where $Y \in L, Y$ is $\mu$ large, $Y \subset X_{n}$, and $Y$ is least in the standard well ordering of $L$ satisfying the foregoing properties. Let $g$ be the function in $L$ where $g: \omega \rightarrow L^{<\omega}$ and $h(n) \in g(n)$ for all $n$. Let $g^{\prime}(n)=\bigcap g(n) . g^{\prime} \in L, g^{\prime}(n)$ is $\mu$ large, and $g^{\prime}(n) \subset X_{n}$. Let $Y_{n}=\bigcap_{i \leqslant n} g^{\prime}(i)$. The sequence $Y_{0} \supset Y_{1} \supset Y_{2} \supset \ldots$ is in $L$ with each $Y_{i} \mu$ large and $Y_{i} \subset X_{i}$. Since $\mu$ is selective in $L$ there is an increasing $f \in L$ such that for all $n, f(n+1) \subset Y_{f(n)}$. Since $Y_{f(n)} \subset X_{f(n)}, f$ works for the given sequence $X_{0} \supset X_{1} \supset X_{2} \supset \ldots$

Proof of THEOREM 6.1. The hard work for Theorem 6.1 is contained in the following lemma.

LEMMA 1. Let $\mu$ be a nonprincipal ultrafilter and let $T_{i}, i<d$, be trees. Partition $\cup_{n \in \omega} \Pi_{i<d} T_{i}(n)=P \cup Q$. One of the following holds.

(a)

$$
\begin{aligned}
(\exists h)(\forall k)(\exists X \subset \omega \text { large })(\forall m \in X)\left(\exists M \subset \prod_{i<d} T_{i}(m)\right) \\
{[M \subset Q \wedge M \text { is an } h, k \text { matrix }] . }
\end{aligned}
$$

(b)

$$
\begin{aligned}
(\forall n)(\exists X \subset \omega \text { large })(\forall m \in X)\left(\exists M \subset \prod_{i<d} T_{i}(m)\right) & \\
& {[M \subset P \wedge M \text { is an n matrix }] . }
\end{aligned}
$$

Proof of Theorem 6.1 From LeMma 1. Without loss of generality it may be assumed that alternative (a) of Lemma 1 holds. The argument parallels the proof of Theorem 1 from Theorem 2.1 given in $\$ 2$.

For each $k$ let $X_{k}$ be a large set such that for each $m \in X_{k}, Q \cap \prod_{i<d} T_{i}(m)$ includes an $h, k+1-h$ matrix. Without loss of generality it may be assumed that $X_{0} \supset X_{1} \supset X_{2} \supset \ldots$ By the selectivity of $\mu$ there is a function $f: \omega \rightarrow \omega$ increasing with large range such that $f(k+1) \in X_{f(k)}$. Let $X$ denote the range of $f$. It follows that if $m \in X$ and $S(X, m)$ is the $X$-successor of $m$ then $S(X, m) \in X_{m}$ i.e. $Q \cap \Pi_{i<d} T_{i}(s(X, m))$ includes an $h, m+1-h$ matrix. $X$ is, in the notation of $\S 2$, a large set of tree levels for the partition.

Following the proof of Theorem 1 from Theorem 2.1 one next obtains a large set of tree levels for the partition all of whose matrices are centered on a single $\vec{a} \in \Pi_{i<d} T_{i}(h)$. Finally, by passing to the appropriate large $Z$ one can be assured that $\vec{a} \in Q$. One then constructs the trees with levels from the large set $Z$ as required for Theorem 6.1 .

It should be remarked that the selectivity of $\mu$ enters only in the construction of $X$. If it can be eliminated there one can weaken the hypotheses of Theorem 6.1. 
Proof of Lemma 1. This argument parallels that of Halpern and Lauchli [4] except that one also keeps track of the nonprincipal ultrafilter $\mu$. Recall from [4] that a $d$ word is a sequence of symbols with length $2 d$. The possible symbols in a $d$ word are $\forall a_{i}, \exists A_{i}, \forall x_{i}, \exists x_{i}$ where $i<d$. The following rules are obeyed in a $d$ word.

(a) $\forall a_{i}$ and $\exists x_{i}$ appear in a $d$ word $W$ exactly when $\exists A_{i}$ and $\forall x_{i}$ do not appear.

(b) The occurrence of $\exists A_{i}$ or $\forall a_{i}$ always precedes the occurrence of $\forall x_{i}$ or $\exists x_{i}$.

One writes $W_{1} \vdash W_{2}$, for $d$ words $W_{1}$ and $W_{2}$, when $W_{2}$ can be obtained from $W_{1}$ by a finite sequence of the elementary instances of $W_{1} \vdash W_{2}$ listed below. Notation is as follows. $\alpha$ and $\beta$ stand for $a_{i}, A_{i}$, or $x_{i} . U$ and $V$ stand for sequences of symbols. $\forall_{i<s} \vec{\alpha}$ or $\exists_{i<s} \vec{\alpha}$ stand for $\forall \alpha_{0} \ldots \forall \alpha_{s-1}$ or $\exists \alpha_{0} \ldots \exists \alpha_{s-1}$ when $\vec{\alpha}=$ $\left(\alpha_{0}, \ldots, \alpha_{s-1}\right)$.

(1) $U \forall \alpha \forall \beta V \vdash U \forall \beta \forall \alpha V$.

(2) $U \exists \alpha \exists \beta V \vdash U \exists \beta \exists \alpha V$.

(3) $U \forall a_{i} \exists x_{i} V \vdash U \exists A_{i} \forall x_{i} V$.

(4) $U \exists A_{i} \forall x_{i} V \vdash U \forall a_{i} \exists x_{i} V$.

(5) $\forall_{n \leqslant i<d} a_{\sigma(i)} \exists_{i \leqslant r} A_{\sigma(i)} U \vdash \exists_{i<r} A_{\sigma(i)} \forall_{r \leqslant i<d} a_{\sigma(i)} U$.

The fundamental fact about $\vdash$ is

LEMMA 2 (HALPERN AND LAUCHLI [4]).

$$
\underset{i<d}{\forall} \vec{a} \underset{i<d}{\exists} \vec{x} \vdash \underset{i<d}{\exists} \vec{A} \underset{i<d}{\forall} \vec{X} .
$$

First assume that the following statement (*) holds.

$$
\begin{aligned}
& (\exists n)\left(\exists_{i<d} \vec{n}: n_{i} \leqslant n\right)(\forall p>n)(\forall R \subset \omega \text { large })(\exists S \subset R \text { large }) \\
& (\forall m \in S: m \geqslant p)\left(\exists_{i<d} \vec{B}: B_{i} i s p \text { dense } \wedge B_{i} \subset T_{i}(m)\right) \\
& \left(\exists_{i<d} \vec{a}: a_{i} \in T_{i}\left(n_{i}\right)\right)\left(\forall_{i<d} \vec{x}: x_{i} \in B_{i} \wedge x_{i} \geqslant a_{i}\right)[\vec{x} \in Q] .
\end{aligned}
$$

We will argue that alternative (a) of Lemma 1 holds in consequence.

The $h$ of alternative (a) will be the $n$ of (*). Let $k$ be given. Set $p=h+k$ and $R=\omega$. Let $X$ be the resulting $S$ intersected with $\omega-p$. We must show that $Q \cap \Pi_{i<d} T_{i}(m)$ includes an $(h, k)$ matrix for all $m \in X$.

Let $\vec{B}$ and $\vec{a}$ be as given in (*) for $m \in X$. For each $i<d$ let $b_{i} \in T_{i}(n)$ be such that $b_{i} \geqslant a_{i}$. This is possible since $n_{i} \leqslant n$. Set $A_{i}=\left\{X_{i} \in B_{i}: X_{i} \geqslant b_{i}\right\}$. Since $b_{i} \in T_{i}(n)=T_{i}(h)$ and $B_{i}$ is $p=h+k$ dense it follows that $A_{i}$ is $(h, k)$ dense. $M=\Pi_{i<d} A_{i} \subset \Pi_{i<d} T_{i}(m)$ is an $(h, k)$ matrix. $M \subset Q$ because each $x_{i} \in A_{i}$ satisfies $x_{i} \in B_{i}$ and $x_{i} \geqslant b_{i} \geqslant a_{i}$.

Now assume that the statement (*) is false. It remains only to show that alternative (b) of Lemma 1 holds in consequence. The negation of (*) is the following statement $(-*)$.

$$
\begin{aligned}
& (\forall n)\left(\forall_{i<d} \vec{n}: n_{i} \leqslant n\right)(\exists p>n)(\exists R \subset \omega \text { large })(\forall S \subset R \text { large }) \\
& (\exists m \in S: m \geqslant p)\left(\forall_{i<d} \vec{B}: B_{i} i s \text { dense } \wedge B_{i} \subset T_{i}(m)\right) \\
& \left(\forall_{i<d} \vec{a}: a_{i} \in T_{i}\left(n_{i}\right)\right)\left(\exists_{i<d} \vec{x}: x_{i} \in B_{i} \wedge x_{i} \geqslant a_{i}\right)[\vec{x} \in P] .
\end{aligned}
$$

$(-*)$ can be simplified to the following statement $(* *)$. 


$$
\begin{aligned}
& (\forall n)\left(\forall_{i<d} \vec{n}: n_{i} \leqslant n\right)(\exists p>n)(\exists R \subset \omega-p \text { large })(\forall m \in R) \\
& \left(\forall_{i<d} \vec{B}: B_{i} \text { is } p \text { dense } \wedge B_{i} \subset T_{i}(m)\right)\left(\forall_{i<d} \vec{a}: a_{i} \in T_{i}\left(n_{i}\right)\right) \\
& \left(\exists_{i<d} \vec{x}: x_{i} \in B_{i} \wedge x_{i} \geqslant a_{i}\right)[\vec{x} \in P] .
\end{aligned}
$$

For the $R$ in (**) one should use

$$
\begin{aligned}
\left\{m \in R^{*}-p:\right. & \left(\underset{i<d}{\forall \vec{B}}: B_{i} \text { is } p \text { dense } \wedge B_{i} \subset T_{i}(m)\right) \\
& \left.\left(\underset{i<d}{\forall} \vec{a}: a_{i} \in T_{i}\left(n_{i}\right)\right)\left(\underset{i<d}{\exists} \vec{x}: x_{i} \in B_{i} \wedge x_{i} \geqslant a_{i}\right)[\vec{x} \in P]\right\}
\end{aligned}
$$

where $R^{*}$ is the $R$ of $(-*)$. This set is large because, according to $(-*)$, it has nonzero intersection with every large set.

In order to prove alternative (b) of Lemma 1 one needs not (**) but the following statement $(* * *)$

$$
\begin{aligned}
& (\forall n)\left(\forall_{i<d} \vec{n}: n_{i} \leqslant n\right)(\exists p>n)(\exists R \subset \omega-p \text { large })(\forall m \in R) \\
& \left(\forall_{i<d} \vec{B}: B_{i} \text { is } p \text { dense } \wedge B_{i} \subset T_{i}(m)\right)\left(\exists_{i<d} \vec{A}: A_{i} \subset B_{i}, n_{i} \text { dense }\right) \\
& \left(\forall_{i<d} \vec{x}: x_{i} \in A_{i}\right)[\vec{x} \in P] .
\end{aligned}
$$

To derive alternative (b) from $(* * *)$ let $n$ be as given in alternative (b), let $\vec{n}=(n, n, \ldots, n)$, and let $X$ be the $R$ from $(* * *)$. For each $m \in X$ let $B_{i}=T_{i}(m)$, all $i<d$ and let $A_{i}$ be as obtained in (***). $\Pi_{i<d} A_{i}$ is an $n$ matrix and, according to (***), $\Pi_{i<d} A_{i} \subset P$.

Thus Lemma 1 has been reduced to proving (***) from $(* *)$. This is where the $d$ words are used. For each $d$ word $W$ let $\Phi(W)$ be the statement

$$
\begin{aligned}
& (\forall n)\left(\forall_{i<d} \vec{n}: n_{i} \leqslant n\right)(\exists p>n)(\exists R \subset \omega-p \text { large })(\forall m \in R) \\
& \left(\forall \forall_{i<d} \vec{B}: B_{i} \text { is } p \text { dense } \wedge B_{i} \subset T_{i}(m)\right) W[\vec{x} \in P]
\end{aligned}
$$

where the quantifiers in $W$ are interpreted as follows.

$\forall a_{i}$ is interpreted as $\left(\forall a_{i} \in T_{i}\left(n_{i}\right)\right)$,

$\exists A_{i}$ is interpreted as $\left(\exists A_{i}: A_{i} \subset B_{i}, n_{i}\right.$ dense),

$\exists x_{i}$ is interpreted as $\left(\exists x_{i}: x_{i} \in B_{i} \wedge x_{i} \geqslant a_{i}\right)$,

$\forall x_{i}$ is interpreted as $\left(\forall x_{i}: x_{i} \in A_{i}\right)$.

These interpretations make sense because of the rules about occurrences of the quantifiers in a $d$ word.

LEMMA 3. If $W_{1} \vdash W_{2}$ then $\Phi\left(W_{1}\right) \Rightarrow \Phi\left(W_{2}\right)$.

Since $(* *)$ is $\Phi\left(\forall_{i<d} \vec{a} \exists_{i<d} \vec{x}\right)$ and $(* * *)$ is $\Phi\left(\exists_{i<d} \vec{A} \forall_{i<d} \vec{x}\right)$ Lemma 2 and Lemma 3 complete the proof of $(* * *)$ from $(* *)$ and therefore the proof of Lemma 1.

Proof of Lemma 3. It suffices to show the 5 special cases of the lemma corresponding to the 5 defining instances of $\vdash$. Instances 1 and 2 are immediate while instances 3 and 4 amount to the observation that there is an $n_{i}$ dense $A_{i} \subset B_{i}$ such that $\left(\forall x_{i} \in A_{i}\right)[\ldots]$ exactly when for each $a_{i} \in T_{i}\left(n_{i}\right)$ there is an $x_{i} \in B_{i}$, such that $x_{i} \geqslant a_{i} \wedge[\ldots]$. Instance 5 of the definition of $\vdash$ must be treated with some care. 
By renumbering the trees it suffices to assume that $W_{1}=\forall_{r<i<d} \vec{a} \exists_{i<r} \vec{A} U$ and $W_{2}=\exists_{i<r} \vec{A} \forall_{r \leqslant i<d} \vec{a} U$. Let $n$ and $\vec{n}$ be given as in $\Phi\left(W_{2}\right)$. Let $N$ be the cardinality of the product $\Pi_{r \leqslant i<d} T_{i}\left(n_{i}\right)$. For $j<N$ define $p_{j}$ and $R_{j}$ as follows. $p_{0}$ and $R_{0}$ are those produced by $\Phi\left(W_{1}\right)$ for the given $n$ and $\vec{n} . p_{j+1}$ and $R_{j+1}$ are those produced by $\Phi\left(W_{1}\right)$ for $n^{\prime}=p_{j}$ and $\vec{n}$ in which $n_{i}^{\prime}=p_{j}$ when $i<r$ and $n_{i}^{\prime}=n_{i}$ when $r \leqslant i<d$. $p$ is taken to be $p_{N-1}$ while $R$ is taken to be $\bigcap_{j<N} R_{j}$. It must now be verified that

$$
(\forall m \in R)\left(\underset{i<d}{\forall} \vec{B}: B_{i} \text { is } p \text { dense } \wedge B_{i} \subset T_{i}(m)\right)(\underset{i<r}{\exists} \vec{A})(\underset{r<i<d}{\forall} \vec{a}) U[\vec{x} \in P]
$$

holds under the designated interpretations of the quantifiers in $W_{2}$.

Accordingly, let $m \in R$ and $B_{i} \subset T_{i}(m) p$ dense be given for all $i<d . \vec{A}_{j}, j<N$ is an $r$ vector defined by downward induction on $j$. Let $a_{j}: j<N$ be an enumeration of $\Pi_{r \leqslant i<d} T_{i}\left(n_{i}\right) . \vec{A}_{N-1}$ is such that $A_{N-1, i} \subset B_{i}$ is $p_{N-2}$ dense, $i<r$, and $U\left(\vec{B}, \vec{A}_{N-1}, \vec{a}_{N-1}\right)[\vec{x} \in P]$ holds. $\vec{A}_{N-1}$ exists because $m \in R \subset R_{N-1}$ and $p_{N-1}$, $R_{N-1}$ solve $\Phi\left(W_{1}\right)$ for the $\vec{n}$ which is identically $p_{N-2}$ for $i<r$ and agrees with $\vec{n}$ otherwise.

Similarly for $0 \leqslant j<N-1, \vec{A}_{j}$ is such that $A_{j, i} \subset A_{j+1, i}$ is $p_{j-1}$ dense and $U\left(\vec{B}_{j+1}, \vec{A}_{j}, \vec{a}_{j}\right)[\vec{x} \in P]$ holds where $B_{j+1}=A_{j+1, i}$ for $i<r$ and $B_{j+1}=B_{i}$ for $r \leqslant i<d$. As above, $A_{j}$ exists because $m \in R \subset R_{j}$ and $p_{j}, R_{j}$ solve $\Phi\left(W_{1}\right)$ for the $\vec{n}^{\prime}$ which is identically $p_{j-1}$ for $i<r$ and agrees with $\vec{n}$ otherwise.

We remark that if $j<k \leqslant N-1$ then $U\left(\vec{B}_{j+1}, \vec{A}_{j}, \vec{a}_{k}\right)[\vec{x} \in P]$ also holds. If $i<r$ then $x_{i}$ occurs in $U$ bound in the quantifier $\left(\forall x_{i}: x_{i} \in A_{i}\right)$. When this is applied to $A_{j, i}$ instead of $A_{k, i}$ the effect is only to restrict the domain of universal quantification because $A_{j, i} \subset A_{k, i}$. Thus the statement continues to hold. If $r<i<d$ then $x_{i}$ occurs in $U$ bound in the quantifier $\left(\exists x_{i}: x_{i} \in B_{j+1, i} \wedge x_{i} \geqslant a_{i}\right) . B_{j+1, i}=B_{k+1, i}=$ $B_{i}$ for $r \leqslant i<d$ so no change in the domain of quantification occurs.

The construction is varied at the last i.e. $j=0$ step so that $A_{j, i}$ is $n_{i}$ dense. It follows that $\vec{A}_{0}$ satisfies $A_{0, i} \subset B_{i}$ and $U\left(\vec{B}_{1}, \vec{A}_{0}, \vec{a}_{k}\right)[\vec{x} \in P]$ holds for every $0<k$ $<N$. Since $B_{i}$ only occurs in $U$ for $r \leqslant i<d$ one has $U\left(\vec{B}, \vec{A}_{0}, \vec{a}\right)[\vec{x} \in P]$ holds for the original $\vec{B}$ and every $\vec{a} \in \Pi_{r \leqslant i<d} T_{i}\left(n_{i}\right)$.

Therefore using $\vec{A}_{0}$ as the required $\vec{A}$ one has

$$
\underset{i<r}{\exists} \vec{A} \underset{r<i<d}{\forall} \vec{a} U(\vec{B}, \vec{A}, \vec{a})[\vec{x} \in P]
$$

holds. This completes the proof of Lemma 3, hence of Lemma 1, Theorem 6.1, and Theorem 6.

\section{BIBLIOGRAPHY}

1. S. Gregorieff, Combinatorics on ideals and forcing, Ann. Math. Logic 3 (1971), 363-394.

2. J. D. Halpern, Independence of the axiom of choice from the Boolean prime ideal theorem, Fund. Math. 55 (1964), 57-66.

3. Nonstandard combinatorics, Proc. London Math. Soc. 30 (1975), 40-54.

4. J. D. Halpern and H. Lauchli, A partition theorem, Trans. Amer. Math. Soc. 124 (1966), 360-367.

5. J. D. Halpern and A. Levy, The Boolean prime ideal theorem does not imply the axiom of choice, Proc. Sympos. Pure Math., Vol. 13, part I, Amer. Math. Soc., Providence, R. I., 1967, pp. 83-134.

6. T. Jech, Lectures in set theory, Lecture Notes in Math., Vol. 217, Springer-Verlag, Berlin and New York, 1971. 
7. K. Milliken, Some results in Ramsey theory, Doctoral Dissertation, UCLA, 1975.

8. ___ A Ramsey theorem for trees, J. Combin. Theory Ser. A 26 (1979), 215-237.

9. D. Pincus, On the independence of the Kinna Wagner principle, Z. Math. Logik Grundlagen Math. 20 (1974), 503-516.

10. __ Adding dependent choice, Ann. Math. Logic 11 (1977), 105-144.

11. __ Adding dependent choice to the prime ideal theorem, Studies in Logic and Found. Math., Vol. 87, North-Holland, Amsterdam, 1977, pp. 547-565.

12. G. Sacks, Forcing with perfect closed sets, Proc. Sympos. Pure Math., Vol. 13, part I, Amer. Math. Soc., Providence, R. I., 1967, pp. 331-355.

Harvard University Medical School, Cambridge, Massachusetts 02138

Department of Mathematics, University of Alabama in Birmingham, Birmingham, Alabama 35294 